\title{
A level-set based topology optimization using the element connectivity parameterization method
}

\author{
N. P. van Dijk - G. H. Yoon • \\ F. van Keulen · M. Langelaar
}

Received: 5 November 2008 / Revised: 4 January 2010 / Accepted: 8 January 2010 / Published online: 27 February 2010

(C) The Author(s) 2010. This article is published with open access at Springerlink.com

\begin{abstract}
This contribution presents a novel and versatile approach to geometrically nonlinear topology optimization by combining the level-set method with the element connectivity parameterization method or ECP. The combined advantages of both methods open up the possibility to treat a wide range of optimization problems involving complex physical and/or geometrical nonlinearities in a general and elegant manner. The level-set method features shape optimization on a fixed mesh, leading to intrinsically black-andwhite designs. This approach allows a clear description of location and orientation of the interface, whereas topological changes can still be handled easily. A popular concept used in conventional level-set methods is to map the levelset function to volume-fraction design variables for every element of a finite element mesh. The resulting element density variables are then used to scale the Young's modulus in each element using the Ersatz material approach. In this work we employ a modified material interpolation method, in which the element density variables, based on a per-element integration of a regularized Heaviside operator applied to the level-set function, are used as element
\end{abstract}

\footnotetext{
N. P. van Dijk ( $₫)$ · F. van Keulen · M. Langelaar

Delft University of Technology,

Mekelweg 2, 2628 CD Delft, The Netherlands

e-mail: N.P.vanDijk@tudelft.nl

F. van Keulen

e-mail: F.vanKeulen@tudelft.nl

M. Langelaar

e-mail: M.Langelaar@tudelft.nl

G. H. Yoon

KyungPook National University, Daegu, Korea

e-mail: ghy@knu.ac.kr
}

connectivity design variables. The resulting crisp boundary topology optimization method exploits the advantages of ECP in the field of complex nonlinearities and eliminates the need for penalization by the implicit level-set description of the design.

Keywords Topology optimization - Level-set method · Element connectivity parameterization method (ECP) . Geometrical nonlinearities

\section{Introduction}

The level-set method is an approach to topology optimization that uses a flexible implicit description of the material domain. This structural domain is represented by a levelset function whose zero-level contour defines the structural boundary (Allaire et al. 2004; Wang et al. 2003; Wang and Wang 2006). During the optimization the zero-level contour of the level-set function is displaced in a favorable direction based on shape sensitivity analysis, while the implicit description of the interface allows for topological changes of the zero-level contour. This enables topology optimization for crisp boundary designs, where the intermediate densities are restricted to a small band around the boundary of the structure.

The general framework of shape optimization gives the level-set method a wide range of applicability. Also, as opposed to other types of topology optimization, the location of (or the distance to) the interface can be retrieved as well as the direction of the normal to this interface (Allaire et al. 2004; Wang et al. 2003; Wang and Wang 2006). This opens up the possibility of topology optimization of design problems involving, for instance, design-dependent forces, e.g. pressure loads (Allaire et al. 2004). Moreover, the 
structural domain is described by a level-set function which ensures a well-defined structural domain, which is free from numerical artifacts.

Many variations to the original immersed interface method (Sethian and Wiegmann 2000) have been proposed, e.g. Allaire et al. (2004), Wang et al. (2003), Wang and Wang (2006). Parts of these variations and techniques from other fields of optimization can be combined in order to obtain efficient and practical approaches to topology optimization. The general structure of the level-set method can be divided into three components; the level-set function parameterization, the level-set function update and the discretization of the governing equations. The approach to each of these components can be decided upon independently. By changing the appropriate components for a specific optimization problem an effective new variant of the level-set family can be obtained.

The level-set method has been extended to the field of nonlinearities, for instance in Allaire et al. (2004). However, physical and geometrical nonlinearities can pose problems for level-set-based as well as density-based topology optimization. In order to deal with these complex nonlinearities in a general and elegant way, the proposed method in this research employs an alternative approach to the discretization of the structural analysis. We combine the level-set method with the element connectivity parametrization method (ECP) (Langelaar 2006; Yoon and Kim 2005a, b; Yoon et al. 2005, 2008). The material domain is now mapped to the finite element mesh employing a modified material interpolation method, in which the element density variables are used as element connectivity design variables. This combination allows an easy treatment of the nonlinearities in a level-set-based topology optimization.

In Section 2 the general structure of the level-set method is treated. Different approaches that can be chosen for individual parts of this structure are discussed. One of these choices involves the discretization of the structural problem. The ECP method that is used as an alternative material interpolation method is treated in Section 3. The framework of an optimization with a general objective is formulated using the proposed combination of the level-set method with ECP in Section 4. For specific choices of the general objective we study the formulation of linear compliance minimization and the geometrically nonlinear compliant mechanism design problems. Then, the novel ECP-based level-set method is validated on the basis of established linear compliance minimization problems in Section 5. The effectiveness and numerical robustness of the coupled method is subsequently demonstrated for geometrically nonlinear compliant mechanism design problems in Section 6. This paper ends with conclusions regarding the proposed method. The detailed derivation of the normal boundary velocity field is documented in Appendix.

\section{Level-set method}

As mentioned, the structure of a level-set method can be divided into the level-set function parameterization, the level-set function update (incorporating sensitivity information) and the discretization of the governing equations. Each of these components will be treated next and the adopted approaches in this research will be indicated.

\subsection{Level-set function parameterization}

The choice of the parameterization of the level-set method defines the design space in which the optimal configuration is sought. The shape of the material domain $\Omega$ with boundary $\Gamma$ in terms of a level-set function $\Phi(\mathbf{x})$ is given as,

$\Phi(\mathbf{x}) \geq 0$ for $\mathbf{x} \in \Omega$

$\Phi(\mathbf{x})<0$ for $\mathbf{x} \in D \backslash \Omega$

$\Phi(\mathbf{x})=0$ for $\mathbf{x} \in \Gamma$,

where $D$ is a large domain fully containing the material domain $\Omega$. An example of a level-set function and the corresponding material domain are displayed in Fig. 1.

A level-set function can be parameterized in terms of any type of basis functions in terms of the spatial coordinates $\mathbf{x}$ and the temporal design parameter $t$. Generally the level-set function $\Phi(\mathbf{x}, t)$ can be written as,

$\Phi(\mathbf{x}, t)=\sum_{i} \beta_{i}(t) \phi_{i}(\mathbf{x})$

where the $\beta_{i}$ 's are the parameters and the $\phi_{i}$ 's are the basis functions. Linear basis functions, as commonly used in finite element discretization, can be used leading to a so called discrete level-set method (Allaire et al. 2004; Wang et al. 2003). However, any other type of basis can be used such as higher-order basis functions or (overlapping) radial basis functions. The latter have been used in some of the

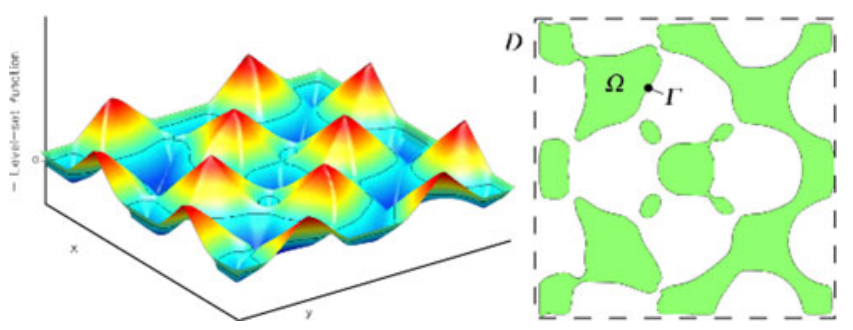

Fig. 1 On the left: an example of a level-set function on a two dimensional domain (the $\mathrm{z}$-axis points downward). On the right: the corresponding zero-level contour of the level-set function or material domain 
proposed parameterized level-set methods (Wang and Wang 2006). In this paper we interpolate the level-set function with linear basis functions with one level-set parameter per finite element.

\subsection{Discretization}

In order to perform a structural optimization, the responses of a design and the sensitivities to a design change need to be calculated using a finite element analysis. For instance, a conforming mesh can be constructed. Such a mesh, with element boundaries coinciding with the boundary of the material domain, provides an accurate solution to the structural problem. This conforming mesh has to be reconstructed for each of the changing designs in the course of the optimization.

On the other hand, one can also opt for a fixed, nonconforming and regular mesh. Instead of constructing a conforming mesh, the level-set function is translated to a non-conforming mesh using a Heaviside operator on the level-set. Computational expenses associated with remeshing procedures can then be avoided, but the results of this type of finite element analysis will be less accurate. This popular and flexible approach is used in this research.

The level-set method, which is formulated in a shape optimization framework, is used as a topology optimization method. Shape sensitivities indicate changes of the objective and constraints due to infinitesimal shape changes. Therefore, the sensitivities may not predict the changes of the responses accurately when topological changes occur. Pure shape sensitivities do not perceive possible favorable connections or unfavorable loss of connections between material regions. This is problematic when the structural problem is solved on a conforming mesh and void regions are not discretized.

Therefore, and because of the computational cost and implementational effort involved with remeshing procedures, most level-set based topology optimization methods, including the method proposed in this paper, use a nonconforming fixed mesh.

In order to perform a finite element analysis on a nonconforming mesh, the level-set function needs to be mapped to a field of element densities $\rho_{e}$. These element densities are usually averaged values of a Heaviside function $H$ applied on the level-set function $\Phi$ per element,

$\rho_{e}=\frac{\int_{\Omega_{e}} H(\Phi) d \Omega}{\int_{\Omega_{e}} d \Omega}$

where $\Omega_{e}$ is the material domain of element $e$. If desired an approximate Heaviside can be used in (5) provided that the level-set function is required to have the signed- distance property. An exact or many forms of an approximate Heaviside can be chosen, for instance the third order polynomial used here, given by,

$$
\begin{cases}H(\Phi)=\varepsilon & \text { for } \Phi<-h \\ H(\Phi)=a\left(\frac{\Phi}{h}\right)^{3}+b \frac{\Phi}{h}+c & \text { for }-h \geq \Phi \geq h \\ H(\Phi)=1 & \text { for } \Phi>h,\end{cases}
$$

where $a=\frac{1}{4}(\varepsilon-1), b=\frac{3}{4}(1-\varepsilon), c=\frac{1}{2}(\varepsilon+1)$, $\varepsilon>0$ is a lower bound for the element densities and $h$ is the bandwidth of the approximate Heaviside. When $h \rightarrow 0$ (6) reduces to an exact Heaviside for the element densities, which corresponds to the material volume fraction per element. An exact Heaviside, as used in i.e. Allaire et al. (2004), results in the most crisp description of an design when using a non-conforming mesh. In Fig. 2 an example of such an element density field (with a bandwidth $h$ of two times the diagonal of a finite element) has been displayed. The configuration is the result of a linear compliance minimization benchmark problem.

This approach generates a topology in which intermediate densities $\varepsilon<\rho<1$ are restricted to a band around the zero-level contour of the level-set function (even when an 'exact' Heaviside is used). This allows for relaxed topologies as an intermediate shape between connected and disconnected features in a design.

The densities $\rho_{e}$ now indicate which finite elements belong (partially) to the material domain and which do not. These densities can be used to directly scale the stiffness of the material, known as the "Ersatz material" approach. The contribution of a single element to the global equilibrium equations for linear elasticity using the Ersatz material approach is given by,

$\rho_{e} \mathbf{K}_{e} \mathbf{U}=\mathbf{F}$,

where $\mathbf{K}_{e}$ is the original element stiffness matrix, $\mathbf{U}$ are the element degrees of freedom and $\mathbf{F}$ is the force vector associated with element $e$.

As mentioned, this paper presents an alternative procedure where the elements are (partially) disconnected from their neighbors when they (partially) do not belong to the

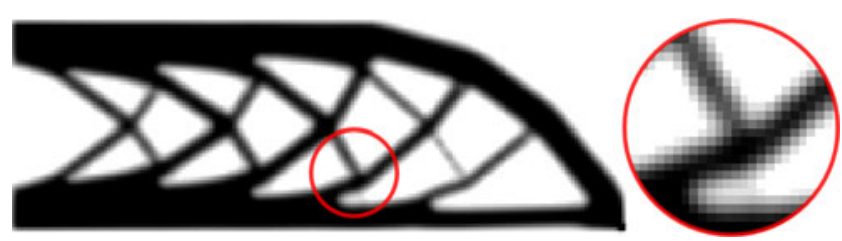

Fig. 2 Element densities of the final design of a linear compliance minimization problem. The bandwidth $h$ used to compute the element densities, equals two times the diagonal of a finite element 
material domain. This approach, called the element connectivity parameterization method (ECP), has the ability to deal with both material and geometrical nonlinearities in a general and elegant way (Yoon and Kim 2005a). The ECP method is treated in more detail in Section 3.

\subsection{Level-set function update}

The level-set function is updated using a boundary design velocity field derived using shape sensitivity analysis. This boundary design velocity is derived in a continuum formulation from the governing equations. The ingredients needed are the structural displacements, the adjoint displacements that enforce the equilibrium equation and, in the case of constrained optimization problems possibly other Lagrange multipliers.

The shape sensitivities are based on boundary displacements over an infinitesimal distance. An infinitesimal boundary displacement in the tangential direction does not change the shape of the design (Allaire et al. 2004; Wang et al. 2003; Wang and Wang 2006). Therefore, an infinitesimal shape change only depends on a displacement in the normal direction. The zero-level contour of the levelset function $\Phi(\mathbf{x}, t)=0$ is a function of the spatial coordinates $\mathbf{x}$ and a design-time $t$ tracking the design changes. The level-set function is convected solving the HamiltonJacobi transport equation on this velocity field derived from the derivative of this contour with respect to the pseudo-time parameter $t$ (Allaire et al. 2004),

$0=\frac{d \Phi(\mathbf{x}, t)}{d t}=\frac{\partial \Phi}{\partial t}+\nabla \Phi \cdot \frac{d \mathbf{x}}{d t}=\frac{\partial \Phi}{\partial t}+\|\nabla \Phi\| v_{\mathbf{n}}(\mathbf{x})$,

where $d \mathbf{x} / d t=v_{\mathbf{n}} \mathbf{n}$ is the design boundary velocity obtained from shape sensitivity analysis. The size of the shape change of the domain can be controlled by scaling the velocity field $v_{\mathbf{n}}$ and/or the total pseudo-time $t$ over which (8) is integrated.

The derivation of the design boundary velocity $v_{\mathbf{n}}$ involves an expression that is evaluated at the boundary. In many cases one can improve the performance of the levelset method by extending this velocity field to a band around the boundary or even throughout the whole domain. This can be done by employing a velocity extension algorithm, extending the normal velocity at the boundary such that it is constant along the normal to the boundary and/or by including regularization to increase the efficiency even further (de Gournay 2006). It is also possible to use a 'natural' extension; evaluating the expression, which is valid at the boundary, everywhere throughout the domain. In this research we use this approach. In Fig. 3 an example of a 'naturally' extended velocity field is shown for a linear compliance minimization problem.

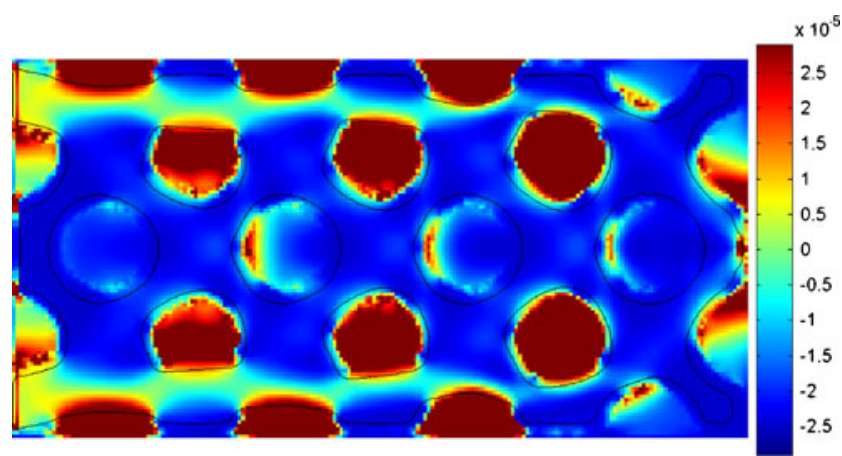

Fig. 3 An example of a 'naturally' extended velocity field of the design optimization of a linear compliance minimization problem. The red areas indicate a positive normal boundary velocity where the material domain will expand and the blue areas indicate a negative velocity where it will shrink

The obtained velocity field is used to update the levelset function in a band around the boundary or on the whole domain. Level-set methods using a regular rectangular grid often use an upwind scheme to update the level-set function (Allaire et al. 2004; Wang et al. 2003; Sethian 1999). Upwind schemes are computationally cheap explicit time integration methods to solve transport equations. They are commonly used to obtain an initial guess in computational fluid dynamics. This type of method, including the proposed method in this paper, is usually referred to as the discrete level-set method.

The result of the upwind procedure slowly approaches a step-like function and destroys the smoothness of the levelset function. In order to accurately approximate the gradient of level-set function (related to the normal and the curvature of the boundary) it is necessary to ensure a sufficiently smooth level-set function or restrict the magnitude of the norm of the gradient. This is usually done by reinitializing the level-set function to a signed-distance function (Allaire et al. 2004; Wang et al. 2003). The signed-distance function also facilitates an easy construction of an approximate Heaviside density field.

Another method is solving the Jacobi-Hamilton equation by translating the global transport equation to a series of ordinary differential equations and solving this system for the coefficients associated with the basis functions of the level-set function parameterization (Wang and Wang 2006),

$0=\frac{\partial \beta_{\mathbf{i}}}{\partial \mathbf{t}} \phi_{\mathbf{i}}-\left\|\sum_{\mathbf{i}} \beta_{\mathbf{i}} \nabla \phi_{\mathbf{i}}\right\| \mathbf{v}_{\mathbf{n}}(\mathbf{x})$.

By choosing radial basis functions in combination with an exact Heaviside, the smoothness of the level-set function may be retained. Therefore, reinitialization procedures are 
not necessary for this kind of level-set update. However, as a consequence the distance to the boundary is unknown and an approximate Heaviside cannot be employed.

Wang and Wang (2006) reports that in this step, any type of optimization algorithm can be used to update the coefficients according to (9) and multiple constraints can be incorporated at the level of the optimizer. A level-set method using this procedure is usually referred to as the parameterized level-set method.

When the level-set function is updated everywhere in the domain and a reinitialization scheme is not employed, the 'natural' velocity extension has the possibility to create new holes in the interior of the material domain (but no approximate Heaviside can be used). In this case the level-set function parameterization should only allow smooth solutions and non-zero gradients almost everywhere in order to avoid numerical artifacts.

An alternative to create new holes is provided by the definition of topological derivatives. A topological derivative provides information on an infinitesimal variation of an (objective) functional when a small hole is created somewhere in the material domain, e.g. Sokolowski and Zochowski (1999), Garreau et al. (2001).

It has been shown that different approaches to each of the separate components of the level-set method can be chosen to obtain a practical and efficient topology optimization algorithm. This paper presents ECP as an alternative to the Ersatz material approach to handle geometrical nonlinearities. The ECP method is treated in the next section.

\section{Element connectivity parameterization method (ECP)}

The common Ersatz material approach in level-set based topology optimization causes a number of difficulties concerning both the convergence of nonlinear analyses as well as that of the optimization process. Bruns and Tortorelli (2003) and Yoon and Kim (2005a) recognized the problem that elements associated with the void region can cause unstable convergence behavior of the analysis. As can be seen in Fig. 4, elements can be distorted to such an extent that the tangent stiffness matrix loses positive definiteness.

Difficulties can also arise when physical nonlinearities are involved (Langelaar 2006; Yoon and Kim 2005b). Nonlinear material models require interpolation schemes to relate intermediate densities to appropriate internal forces. For each type of nonlinear behavior different schemes have to be devised in order to obtain a functional optimization algorithm. For complicated nonlinear models a functioning interpolation scheme is often hard or impossible to find and the optimization process will have severe convergence problems.

Recently, ECP has been developed as an alternative to the Ersatz material approach (direct scaling of the stiffness e.g. SIMP) (Langelaar 2006; Yoon and Kim 2005a, b; Yoon et al. 2005). In this new approach all void elements have the ability to 'slide' through each other while retaining their original stiffness. Therefore, the positive definiteness of the tangent stiffness matrix will remain unaffected. In Fig. 5 it can be seen how 'void' elements slide over each other.

In order to obtain this behavior (internal) ECP does not scale the Young's modulus of a finite element according to the element densities (Yoon et al. 2005, 2008). Instead, all elements are disconnected from each other and reconnected to an intermediate displacement field using linear zero-length springs. Thus, we have created a new finite element patch consisting of internal and external degrees of freedom, zero-length linear springs and the original internal element, see Fig. 6. It is now possible to disconnect this internal element from its neighbors by letting the stiffness of the springs $k_{l} \rightarrow 0$. On the other hand, letting $k_{l} \rightarrow \infty$ rigidly connects the internal element to the external degrees of freedom. Scaling the spring stiffnesses $k_{l}\left(\rho_{e}\right)$ as a function of $\rho_{e}$, we can now (partially) disconnect elements from
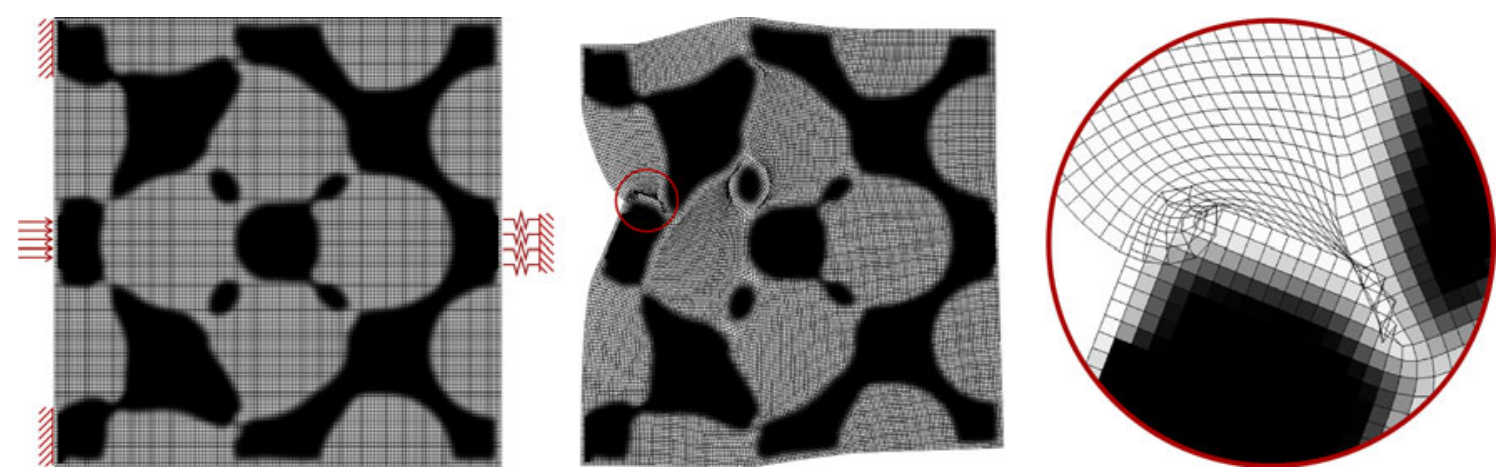

Fig. 4 Severely distorted or inverted void elements can cause instability when using the Ersatz material approach 

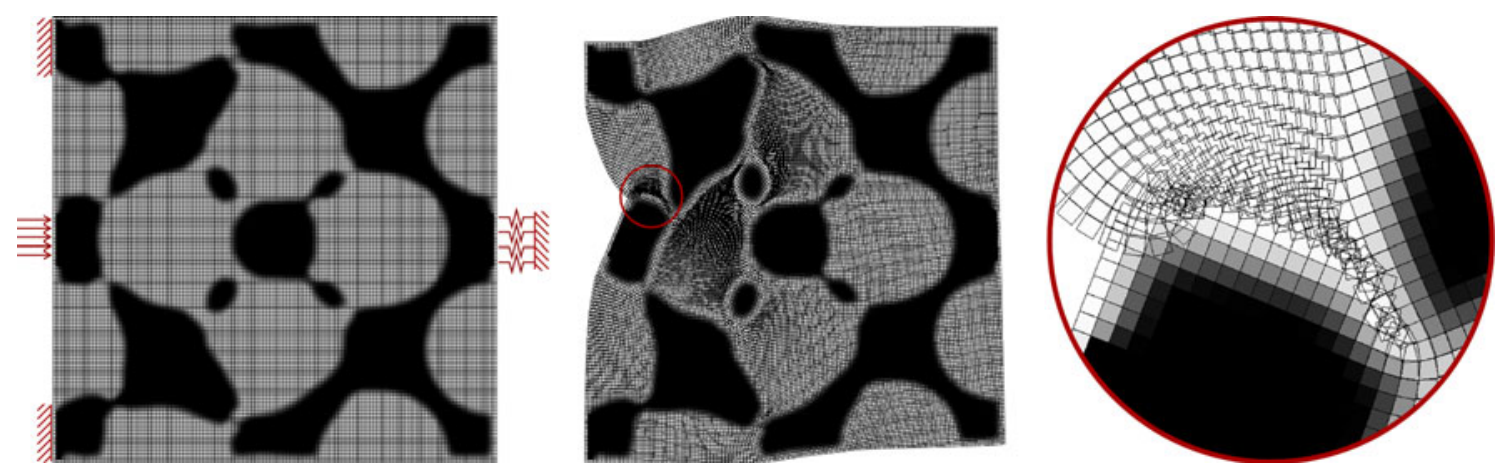

Fig. 5 Disconnected void elements in the ECP method are able to retain the positive definiteness of the tangent stiffness matrix

the mesh, simulating the absence of material. In a linear setting, the contribution of such a single patch (quadrilateral and zero-length linear springs) to the global system can be written as (see Fig. 6),

$\left[\begin{array}{cc}k_{l} \mathbf{I} & -k_{l} \mathbf{I} \\ -k_{l} \mathbf{I} & \mathbf{K}_{e}+k_{l} \mathbf{I}\end{array}\right]\left(\begin{array}{l}\mathbf{U} \\ \mathbf{u}\end{array}\right)=\left(\begin{array}{l}\mathbf{F} \\ \mathbf{0}\end{array}\right)$,

where $\mathbf{K}_{e}$ is the original element stiffness matrix, $\mathbf{I}$ is the unity matrix, $\mathbf{U}$ are the external degrees of freedom, $\mathbf{u}$ are the internal degrees of freedom and $\mathbf{F}$ are the external forces. The introduction of the set of internal degrees of freedom $\mathbf{u}$ increases the size of the problem. However, the external degrees of freedom $\mathbf{U}$ are globally shared degrees of freedom, whereas the internal degrees of freedom $\mathbf{u}$ are only internal degrees of freedom and not shared with other elements. Therefore, the internal stiffness of the original quadrilateral can be condensed onto the external degrees of freedom in order to reduce the size of the problem and keep only the external degrees of freedom as variables,

$$
\begin{aligned}
\mathbf{u} & =k_{l}\left[\mathbf{K}_{e}+k_{l} \mathbf{I}\right]^{-1} \mathbf{U} \\
& \Rightarrow\left[k_{l} \mathbf{I}-k_{l}^{2}\left[\mathbf{K}_{e}+k_{l} \mathbf{I}\right]^{-1}\right] \mathbf{U}=\mathbf{K}_{c} \mathbf{U}=\mathbf{F} .
\end{aligned}
$$

In Yoon et al. (2008) it is shown that for $k_{l} \rightarrow \infty$ the condensed stiffness of the patch goes to the original stiffness, $\mathbf{K}_{c} \rightarrow \mathbf{K}_{e}$, and for $k_{l} \rightarrow 0$ to zero, $\mathbf{K}_{c} \rightarrow \mathbf{0}$, as expected. It is possible to use the link stiffnesses $k_{l}$ as design variables

Fig. 6 Definition of an ECP patch with an internal element with stiffness $\mathbf{K}_{e}$ and connecting zero-length springs with stiffness $k_{l}$

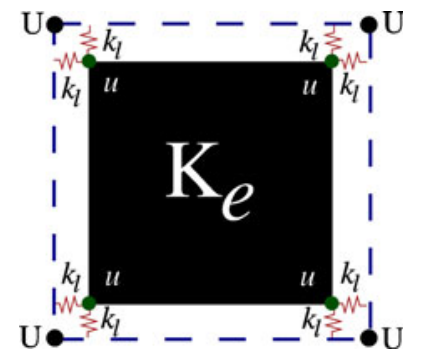

in an optimization. However, the resulting relation between the overall stiffness $\left|\mathbf{K}_{c}\right|$ and $k_{l}$ has become highly nonlinear (though still strictly monotonic), where $|\cdot|$ represents the largest eigenvalue matrix norm. Realizing that the zerolength spring stiffnesses $k_{l}$ are functions of the element densities $\rho_{e}$, we can scale this relation such that the resulting condensed matrix $\mathbf{K}_{c}\left(k_{l}\right)$ scales linearly with $\rho_{e}$ (Langelaar 2006; Yoon et al. 2008),

$\left|\mathbf{K}_{c}\left(k_{l}\left(\rho_{e}\right)\right)\right| \propto \rho_{e}$.

For more detailed information about the exact mathematics the reader is referred to Langelaar (2006), Yoon et al. (2008).

Note that the link stiffnesses are bounded, $0<k_{l}<\infty$ as well as the element densities $\epsilon \leq \rho_{e} \leq 1$, to ensure a well-conditioned, positive definite stiffness matrix of the global problem and ensure that the inversion in (11) can be carried out.

The same treatment can be applied to the nonlinear problem, which is given by,

$\mathbf{0}=\mathbf{R}(\hat{\mathbf{U}}, \hat{\mathbf{u}})=\mathbf{F}_{\text {ext }}-\mathbf{F}_{\text {int }}(\hat{\mathbf{U}}, \hat{\mathbf{u}})$,

where $\mathbf{R}, \mathbf{F}_{\text {ext }}, \mathbf{F}_{\text {int }}, \hat{\mathbf{U}}$ and $\hat{\mathbf{u}}$ are the residual, the external and internal force vector and the assembled external and internal degrees of freedom, respectively.

In each Newton iteration of a nonlinear problem a linear problem has to be solved (Yoon and Kim 2005b),

$\mathbf{K}\left(\hat{\mathbf{U}}^{s}, \hat{\mathbf{u}}^{s}\right)\left(\begin{array}{c}\Delta \hat{\mathbf{U}}^{s+1} \\ \Delta \hat{\mathbf{u}}^{s+1}\end{array}\right)=\mathbf{R}\left(\hat{\mathbf{U}}^{s}, \hat{\mathbf{u}}^{s}\right)$,

where $\mathbf{K}$ is the global assembled tangent matrix. The contribution of an individual patch to this relation, similar to (11) and (12), is given by,

$\left[\begin{array}{cc}k_{l} \mathbf{I} & -k_{l} \mathbf{I} \\ -k_{l} \mathbf{I} & \mathbf{K}_{e}\left(\mathbf{u}^{s}\right)+k_{l} \mathbf{I}\end{array}\right]\left(\begin{array}{c}\Delta \mathbf{U}^{s+1} \\ \Delta \mathbf{u}^{s+1}\end{array}\right)$
$\quad=\left(\begin{array}{c}\mathbf{F} \\ \mathbf{0}\end{array}\right)-\left(\begin{array}{c}k_{l}\left(\mathbf{U}^{s}-\mathbf{u}^{s}\right) \\ \mathbf{f}_{e}\left(\mathbf{u}^{s}\right)-k_{l}\left(\mathbf{U}^{s}-\mathbf{u}^{s}\right)\end{array}\right)$, 
where the element tangent stiffness matrix $\mathbf{K}_{e}\left(\mathbf{u}^{s}\right)=\mathbf{K}_{e}^{s}$ and the element internal force vector $\mathbf{f}_{e}\left(\mathbf{u}^{s}\right)=\mathbf{f}_{e}^{s}$ are functions of the internal displacement $\mathbf{u}^{s}$. The relation between the link stiffness $k_{l}$ and the element density $\rho_{e}$ is obtained from (13) in the first iteration. The internal displacements only depend on the outer displacements, therefore we can eliminate $\Delta \mathbf{u}^{s+1}$ from the global problem by condensation. After condensation we are left with the following contribution of an individual patch,

$$
\begin{aligned}
\Delta \mathbf{u}^{s+1} & =\left[\mathbf{K}_{e}^{s}+k_{l} \mathbf{I}\right]^{-1}\left(k_{l}\left(\mathbf{U}^{s}-\mathbf{u}^{s}\right)-\mathbf{f}_{e}^{s}+k_{l} \Delta \mathbf{U}^{s+1}\right) \\
& \Rightarrow \mathbf{K}_{c}\left(\mathbf{U}^{s}, \mathbf{u}^{s}\right) \Delta \mathbf{U}^{s+1}=\mathbf{R}_{c}\left(\mathbf{U}^{s}, \mathbf{u}^{s}\right)
\end{aligned}
$$

where,

$$
\begin{aligned}
\mathbf{K}_{c}\left(\mathbf{U}^{s}, \mathbf{u}^{s}\right)= & k_{l} \mathbf{I}-k_{l}^{2}\left[\mathbf{K}_{e}^{s}+k_{l} \mathbf{I}\right]^{-1} \\
\mathbf{R}_{c}\left(\mathbf{U}^{s}, \mathbf{u}^{s}\right)= & \mathbf{F}-k_{l}\left(\mathbf{U}^{s}-\mathbf{u}^{s}\right) \\
& +k_{l}\left[\mathbf{K}_{e}^{s}+k_{l} \mathbf{I}\right]^{-1}\left(k_{l}\left(\mathbf{U}^{s}-\mathbf{u}^{s}\right)-\mathbf{f}_{e}^{s}\right) .
\end{aligned}
$$

Now the external displacement increments $\Delta \mathbf{U}^{s+1}$ can be obtained from a system of the size of the original problem. After solving for the external displacement increment $\Delta \mathbf{U}^{s+1}$ the internal displacement increment $\Delta \mathbf{u}^{s+1}$ can be obtained from (17) in a post processing step.

The structural solution obtained using ECP approaches the solution of the Ersatz-material approach in the material domain, and provides more flexibility in the void regions, particularly useful in areas where void is compressed. Where the Ersatz approach suffers from loss of positive definiteness of the tangent stiffness matrix due to excessive compression of void regions, ECP effectively disconnects the nonlinear elements from the mesh, preventing excessive deformation. For more information on the theoretical aspects of ECP the reader is referred to Yoon et al. (2008).

Next, we proceed to the general formulation of the proposed combination of the level-set method and ECP in Section 4.

\section{Proposed method}

The proposed combination of the level-set method and ECP aims to exploit the advantages of both methods. The usage of a level-set function ensures a clear description of the material domain. Furthermore, the location of the boundary is available as the zero-level contour $\Phi(\mathbf{x})=0$ and the outward normal $\mathbf{n}$ can be computed from $\mathbf{n}=-\nabla \Phi /\|\nabla \Phi\|$. The ECP material interpolation allows including geometrical and physical nonlinearities in an elegant way and improves the stability of the finite element analysis involving intermediate densities greatly.
Structural optimization problems including compliance minimization problems and compliant mechanism designs can be generally formulated as follows. The structural problem is defined on the material domain $\Omega$, having a prescribed traction on the Neumann boundary $\Gamma_{N}$ and a prescribed displacement on the Dirichlet boundary $\Gamma_{D}$. With the assumption that there are no body forces, the minimization problem with general objective $J(\mathbf{v})$ with arguments involving a volume term $j_{\Omega}$ and a boundary term $j_{\Gamma}$, is given by,

$\min _{\Omega} J(\mathbf{v})=\int_{\Omega} j_{\Omega}(\mathbf{v}) d \Omega+\int_{\Gamma} j_{\Gamma}(\mathbf{v}) d \Gamma$

subject to $\left\{\begin{array}{ccc}\nabla \cdot \boldsymbol{\sigma}(\mathbf{v})=\mathbf{0} & \text { on } & \Omega \\ \boldsymbol{\sigma}(\mathbf{v}) \cdot \mathbf{n}=\mathbf{t} & \text { on } & \Gamma_{N} \\ \mathbf{v}=\mathbf{0} & \text { on } & \Gamma_{D},\end{array}\right.$

where $\mathbf{v}$ is the displacement, $\boldsymbol{\sigma}$ is the stress, $\mathbf{n}$ is the normal to the boundary and $\mathbf{t}$ is the boundary traction vector. An additional volume constraint can be added to the constraints formulated as,

$G(\Omega)=\int_{\Omega} d \Omega-V_{\max } \leq 0$

where $V_{\max }$ is the maximum volume. Considering a nondesign-dependent surface traction, we do not allow shape changes on the part of the boundary where $\mathbf{t} \neq \mathbf{0}$. We give the results of the derivation from the augmented Lagrangian formulation of the problem above. Details can be found in Appendix. The resulting velocity field $v_{\mathbf{n}}$ is given by,

$$
\begin{aligned}
v_{\mathbf{n}}=-s & \left(j_{\Omega}+\nabla \mathbf{q}: \boldsymbol{\sigma}(\mathbf{v})\right. \\
& \left.+\nabla \cdot\left[j_{\Gamma} \mathbf{n}\right]+l+\alpha G(\Omega)\right) \quad \text { on } \Omega,
\end{aligned}
$$

where $s>0$ is a scaling parameter, $\mathbf{q}$ is the adjoint displacement, $l$ and $\alpha>0$ are the Lagrange multiplier estimate and the penalty parameter associated with a possibly active volume constraint, respectively. When the volume constraint is inactive, both the Lagrange multiplier estimate $l$ and the penalty parameter $\alpha$ is set to zero. The adjoint displacement $\mathbf{q}$ can be solved from the set of equations (Appendix),

$$
\left\{\begin{array}{ccc}
\nabla \cdot(\partial \boldsymbol{\sigma} / \partial \nabla \mathbf{v}: \nabla \mathbf{q})-\partial j_{\Omega} / \partial \mathbf{v}=\mathbf{0} & \text { on } & \Omega \\
\partial \boldsymbol{\sigma} / \partial \nabla \mathbf{v}: \nabla \mathbf{q} \cdot \mathbf{n}=-\partial j_{\Gamma} / \partial \mathbf{v} & \text { on } & \Gamma_{N} \\
\mathbf{q}=\mathbf{0} & \text { on } & \Gamma_{D},
\end{array}\right.
$$

where $\partial \boldsymbol{\sigma} / \partial \nabla \mathbf{v}$ is the tangent stiffness around the solution of the structural displacement $\mathbf{v}$. This problem is exactly equivalent to a Newton iteration in the solving of the structural equilibrium (22) with different forcing terms. 
The Lagrange multiplier estimate $l$ for an active volume constraint can be obtained from (Appendix),

$l=-\frac{\int_{\Gamma_{0}}\left(j_{\Omega}+\nabla \mathbf{q}: \boldsymbol{\sigma}(\mathbf{v})+\nabla \cdot\left[j_{\Gamma} \mathbf{n}\right]\right) d \Gamma}{\int_{\Gamma_{0}} d \Gamma}$,

where $\Gamma_{0}=\Gamma \backslash \Gamma_{\mathbf{t} \neq \mathbf{0}}$.

The level-set method provides a way to use the shape sensitivities given above in a framework allowing for topological changes. The optimization algorithm using the combination of the level-set method and ECP starts from the definition of an initial topology or material domain $\Omega$ in terms of an initial level-set function $\Phi(\mathbf{x}, 0)$. In order to obtain a flexible description of the structural problem, the structural domain is mapped to a fixed, non-conforming and rectangular finite element mesh using quadrilateral elements. We interpolate the level-set function with linear basis functions, one level-set grid point per finite element, as displayed in Fig. 7.

The element densities $\rho_{e}$ on this mesh are calculated according to (5) with an approximate Heaviside with a bandwidth of two times the length of the diagonal of a finite element and a lower bound of $\varepsilon=1 \cdot 10^{-6}$. Then the ingredients of the boundary design velocity field defined by (24), the structural and the adjoint displacement $\mathbf{v}$ and $\mathbf{q}$, respectively, are obtained by solving the structural and the adjoint problem, (25) and (22), discretized using ECP. In order to obtain a well-conditioned optimization problem, the element densities $\rho_{e}$ are used to scale the element link stiffnesses $k_{l, e}$ to satisfy (13).

The stepsize $s$ in (24) and the total pseudo-time over which (8) is integrated are determined adaptively such that the shape change lies in a range between $0.5 \%$ and $0.75 \%$ of the size of domain $\Omega$. The resulting boundary design velocity field $v_{n}$ is 'naturally' extended to the whole domain $D$ and is used by a first-order upwind scheme to evolve the level-set function in the steepest descent direction. In a next step, the level-set function is reinitialized to the signed distance function with another upwind scheme. This is done to retain the accuracy of the normal or gradient of the level-set function and allow for the definition of a new approximate Heaviside density field. This procedure is repeated until the

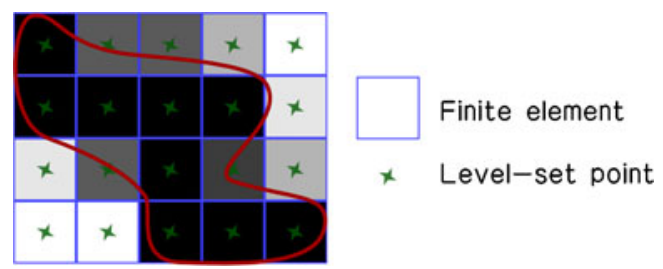

Fig. 7 Discretization of the level-set function with respect to the finite element mesh. The level-set contour $\Phi=0$ is given by the red line and the resulting element densities are indicated by grayscale coloring material domain does not change anymore and a visually 'convergence' is reached.

The proposed method has been implemented for both linear and geometrically nonlinear structural optimization. In Section 5 two linear compliance minimization benchmark problems are treated.

\section{Compliance minimization}

The objective of a linear compliance minimization problem (in absence of a body force) is given by $J(\mathbf{v})=\int_{\Gamma} \mathbf{v} \cdot \mathbf{t} d \Gamma$. We set a volume constraint of $50 \%$ of the total domain,

$G(\Omega)=\int_{\Omega} d \Omega-\frac{1}{2} V_{D} \leq 0$,

where $V_{D}$ is the volume of the total domain $D$ containing the material domain $\Omega$.

For linear elasticity the constitutive relation is given by $\boldsymbol{\sigma}(\mathbf{v})=\mathbf{S}: \boldsymbol{\epsilon}(\mathbf{v})$, where $\mathbf{S}$ is the fourth-order stiffness tensor and $\boldsymbol{\epsilon}(\mathbf{v})=\frac{1}{2}\left(\nabla \mathbf{v}+\nabla \mathbf{v}^{T}\right)$ is the linear strain tensor. The system of equations for the adjoint displacement now reduces to (see Appendix),

$$
\left\{\begin{array}{ccc}
\nabla \cdot(\partial \boldsymbol{\sigma} / \partial \nabla \mathbf{v}: \nabla \mathbf{q})=\nabla \cdot(\mathbf{S}: \nabla \mathbf{q})=\mathbf{0} & \text { on } & \Omega \\
\mathbf{S}: \nabla \mathbf{q} \cdot \mathbf{n}=-\mathbf{t} & \text { on } & \Gamma_{N} \\
\mathbf{q}=\mathbf{0} & \text { on } & \Gamma_{D}
\end{array}\right.
$$

Because of the symmetry of $\mathbf{S}$ we can write,

$$
\left\{\begin{array}{ccc}
\nabla \cdot\left(\mathbf{S}: \frac{1}{2}\left(\nabla \mathbf{q}+\nabla \mathbf{q}^{T}\right)\right)=\nabla \cdot(\mathbf{S}: \boldsymbol{\epsilon}(\mathbf{q}))=\mathbf{0} & \text { on } & \Omega \\
\mathbf{S}: \nabla \mathbf{q} \cdot \mathbf{n}=\mathbf{S}: \boldsymbol{\epsilon}(\mathbf{q}) \cdot \mathbf{n}=-\mathbf{t} & \text { on } & \Gamma_{N} \\
\mathbf{q}=\mathbf{0} & \text { on } & \Gamma_{D} .
\end{array}\right.
$$

Comparing, the structural displacement is solved from,

$$
\left\{\begin{array}{clc}
\nabla \cdot \boldsymbol{\sigma}(\mathbf{v})=\nabla \cdot(\mathbf{S}: \boldsymbol{\epsilon}(\mathbf{v}))=\mathbf{0} & \text { on } & \Omega \\
\boldsymbol{\sigma}(\mathbf{v}) \cdot \mathbf{n}=\mathbf{S}: \boldsymbol{\epsilon}(\mathbf{v}) \cdot \mathbf{n}=\mathbf{t} & \text { on } & \Gamma_{N} \\
\mathbf{v}=\mathbf{0} & \text { on } & \Gamma_{D}
\end{array}\right.
$$

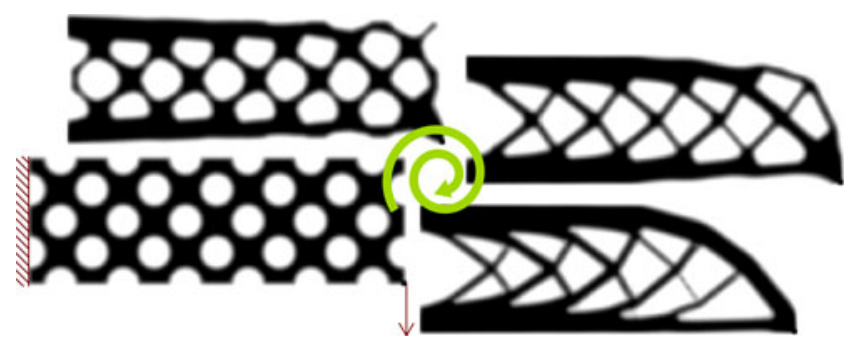

Fig. 8 Initial and evolving element density field during the design optimization of a linear compliance minimization problem using the level-set method combined with ECP 


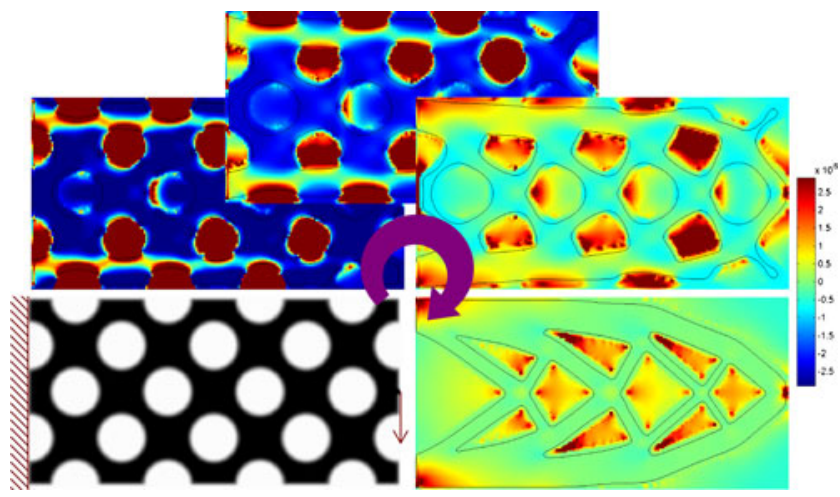

Fig. 9 Initial configuration and the evolving 'naturally' extended velocity field during the design optimization of a linear compliance minimization problem using the level-set method combined with ECP

so for linear elasticity we have $\mathbf{q}=-\mathbf{v}$, associated with the self-adjoint linear compliance minimization problem. This system is cast into a weak form and solved in a finite element analysis as described above. When the volume constraint is active, the Lagrange multiplier estimate $l$ is obtained from,

$l=-\frac{\int_{\Gamma_{0}} \nabla \mathbf{q}: \boldsymbol{\sigma}(\mathbf{v}) d \Gamma}{\int_{\Gamma_{0}} d \Gamma}=\frac{\int_{\Gamma_{0}} \boldsymbol{\epsilon}(\mathbf{v}): \mathbf{S}: \boldsymbol{\epsilon}(\mathbf{v}) d \Gamma}{\int_{\Gamma_{0}} d \Gamma}$,

because $\mathbf{t}=\mathbf{0}$ on $\Gamma_{0}$. A smooth transition from constraint activity to non-activity has been implemented to improve the convergence of the optimization problem. The design velocity is now given by,

$$
\begin{aligned}
v_{\mathbf{n}} & =-s(\nabla \mathbf{q}: \boldsymbol{\sigma}(\mathbf{v})+l+\alpha G(\Omega)) \\
& =s(\boldsymbol{\epsilon}(\mathbf{v}): \sigma(\mathbf{v})-l-\alpha G(\Omega)) \quad \text { on } \Omega,
\end{aligned}
$$

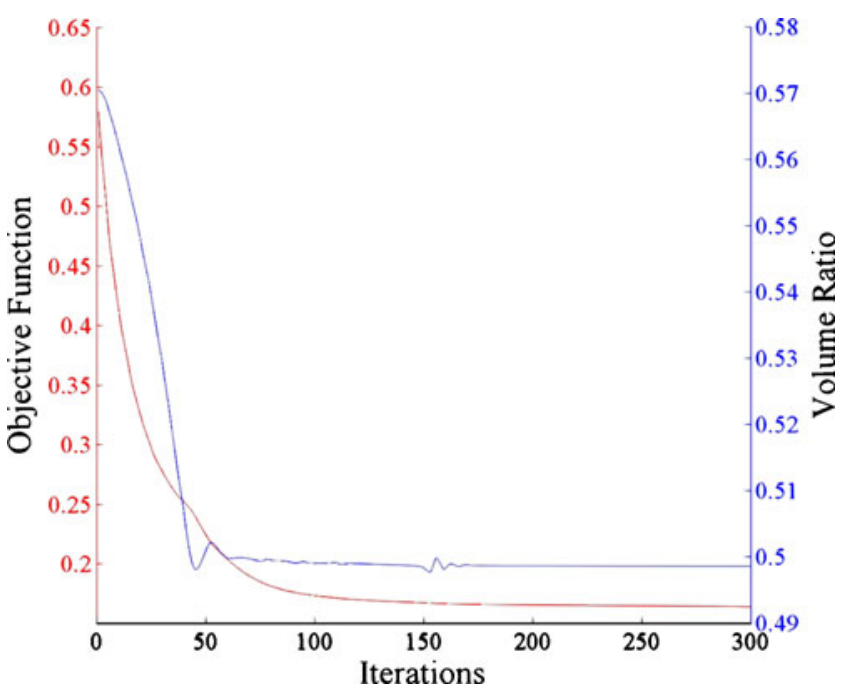

Fig. 10 Objective and constraint history (in \% of the total domain $D$ ) of the design optimization of the second linear compliance minimization problem using the level-set method combined with ECP
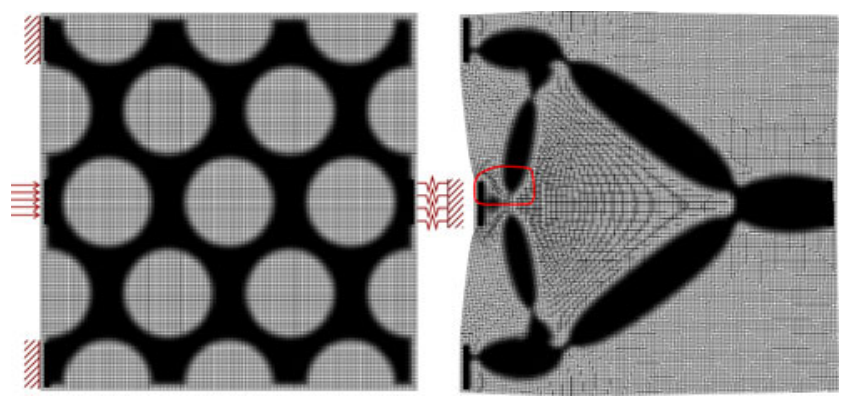

Fig. 11 Plot of the element densities of the initial and optimized deformed configuration of the geometrically nonlinear compliant force inverter using the level-set method combined with ECP. A close-up is shown in the next figure

where $\alpha=\frac{1}{10} A_{e}$ is a volume penalty parameter, chosen as a fraction of the element area $A_{e}$.

One of the most common benchmark problems for topology optimization is the compliance minimization of a cantilever beam supported at one end and loaded at the bottom of the other end in the vertical direction. This benchmark problem has been solved using a domain of $2 \times 1$ meshed with $(80+2) \times(40+4)$ elements; on the free edges of the domain (upper, lower and right side) a row of two elements was added. The Young's modulus and Poisson's ratio are 1,000 and 0.3 , respectively, and the applied force is 1 . As can be seen in Fig. 8, the classical result is obtained using the level-set method in combination with ECP.

Also a problem involving a cantilever loaded vertically at the center of one its end has been solved. This time the domain of $3 \times 1$ is meshed with $(180+2) \times(60+4)$ elements. The Young's modulus, Poisson's ratio and the force remain the same. The results of this benchmark problem are shown in Fig. 9. The convergence behavior of this second optimization, displayed in Fig. 10, is rather smooth.

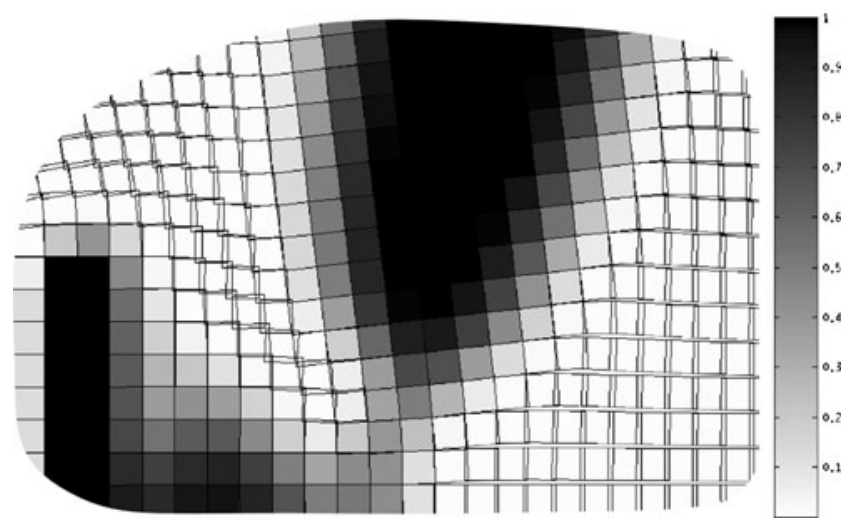

Fig. 12 Close-up of the element densities in the optimized deformed configuration of the geometrically nonlinear compliant force inverter using the level-set method combined with ECP 


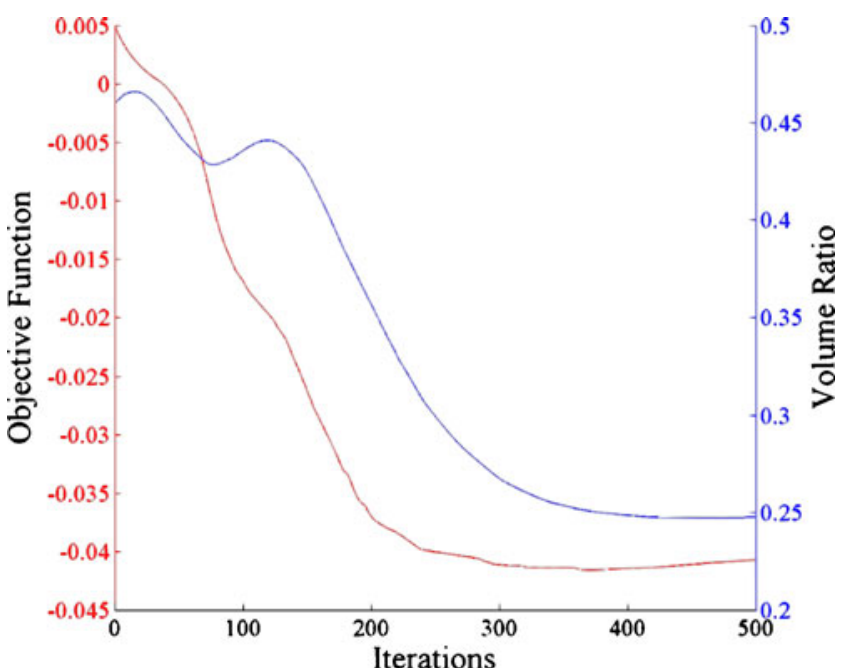

Fig. 13 Objective history and volume fraction history of the geometrically nonlinear compliant force inverter design optimization

\section{Compliant mechanisms}

To demonstrate the effectiveness of the proposed combination of the level-set method and ECP we present two types of complaint mechanism design benchmark problems. Both a compliant force inverter and a compliant gripper have been optimized. The optimized displacement at an internal point of the material domain can be expressed as $\mathbf{L}(\mathbf{x}) \cdot \mathbf{v}$ where $\mathbf{L}(\mathbf{x})$ is an operator selecting the part of the structure with the desired displacement. The objective of the optimization problem is now given by,

$$
J(\mathbf{v})=\int_{\Omega} \mathbf{L} \cdot \mathbf{v} d \Omega .
$$

The adjoint displacement can be now solved from the set of equations (see Appendix),

$$
\left\{\begin{array}{ccc}
\nabla \cdot(\partial \boldsymbol{\sigma} / \partial \nabla \mathbf{v}: \nabla \mathbf{q})-\mathbf{L}=\mathbf{0} & \text { on } & \Omega \\
\partial \boldsymbol{\sigma} / \partial \nabla \mathbf{v}: \nabla \mathbf{q} \cdot \mathbf{n}=\mathbf{0} & \text { on } & \Gamma_{N} \\
\mathbf{q}=\mathbf{0} & \text { on } & \Gamma_{D},
\end{array}\right.
$$

where $\partial \boldsymbol{\sigma} / \partial \nabla \mathbf{v}$ is the tangent stiffness around the solution of the structural displacement $\mathbf{v}$. In this nonlinear setting the adjoint can be found relatively cheaply by solving a linear problem involving the tangent stiffness $\partial \boldsymbol{\sigma} / \partial \nabla \mathbf{v}$. The resulting velocity field $v_{\mathbf{n}}$ for this type of problems is given by,

$v_{\mathbf{n}}=-s(\mathbf{L} \cdot \mathbf{v}+\nabla \mathbf{q}: \sigma(\mathbf{v})+\alpha) \quad$ on $\Omega$,

where $\alpha$ is a very small volume penalty parameter to eliminate unnecessary material (as if the term in the integral of (33) were $[\mathbf{L} \cdot \mathbf{v}+\alpha])$.

The compliant force inverter is meshed with $125 \times 125$ elements in a $1 \times 1$ domain. The Young's modulus equals 1,000 , the Poison's ratio equals 0.3 and the total applied force equals 1 . The boundary conditions and the initial and the optimal configuration, very similar to the one obtained
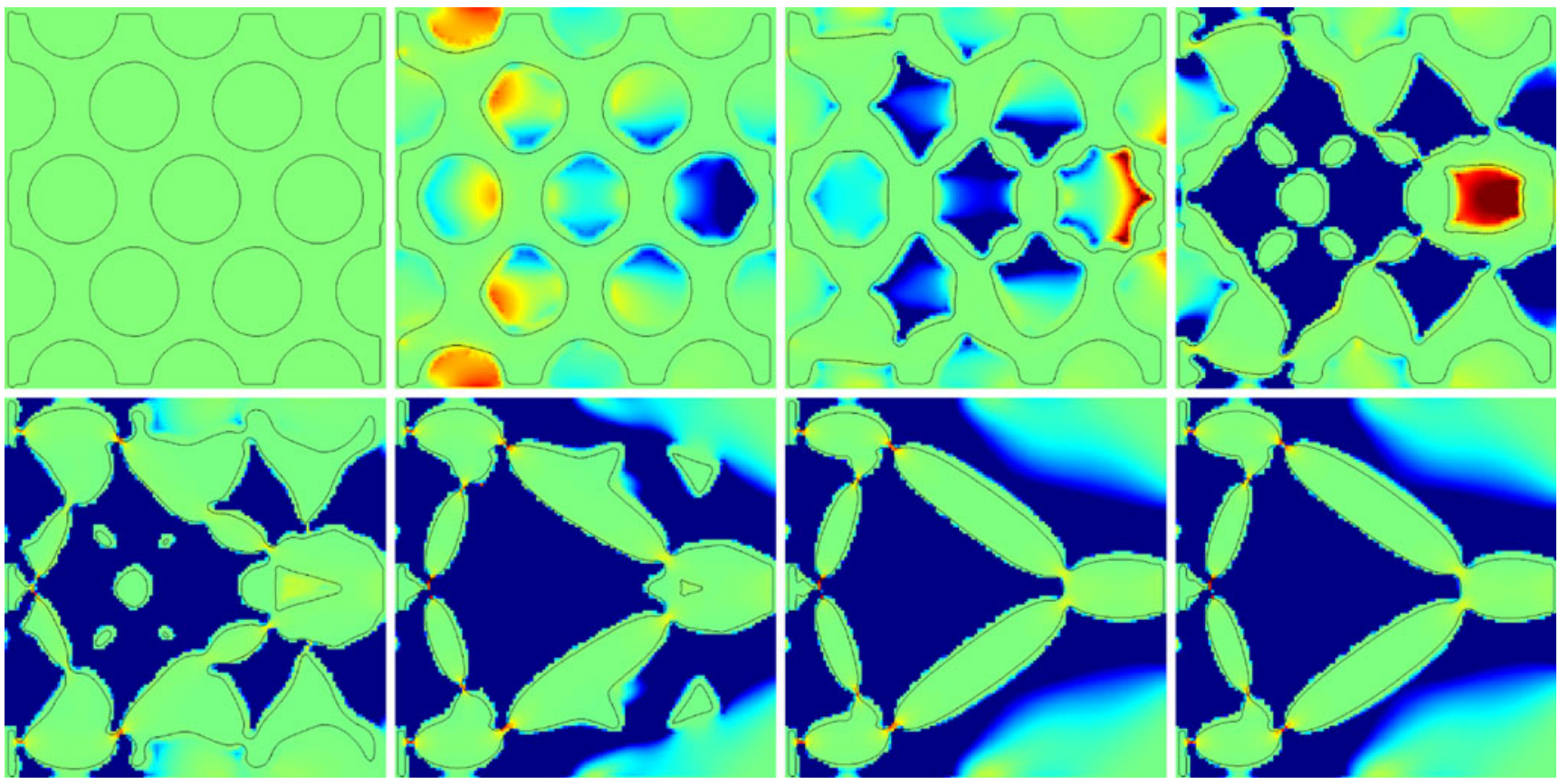

Fig. 14 Design evolution of the 'naturally' extended velocity field of the geometrically nonlinear compliant force inverter design optimization problem. The red areas have a positive design velocity in the direction of the outward normal to the boundary and the blue areas a negative one 


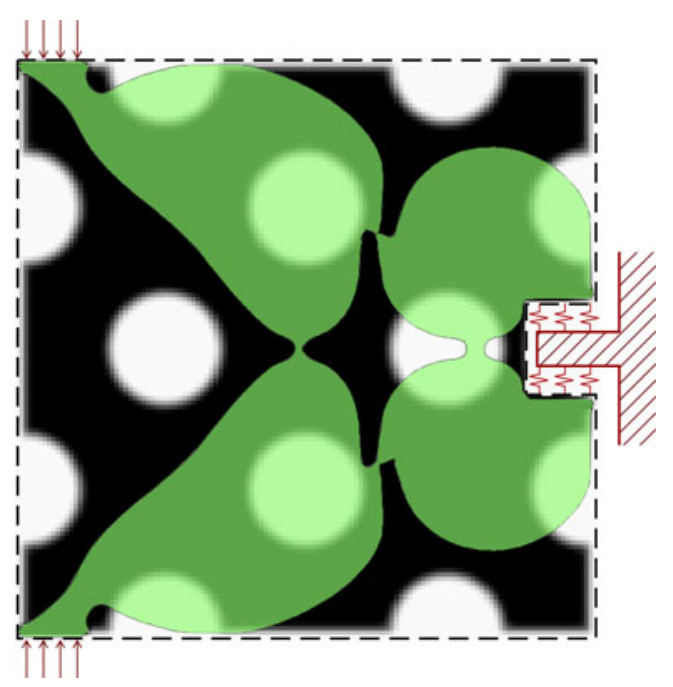

Fig. 15 Plot of element densities of the initial configuration of a compliant gripper design problem and the opaque image of the optimal structural domain obtained using the level set method combined with ECP

in Luo and Tong (2008), are shown in Fig. 11. A close-up of the internal displacements can be seen in Fig. 12. A distributed spring with a stiffness of half the Young's modulus times the height of the structure is attached to the structure to represent the stiffness of an attached structure. The optimum that is reached depends on the choice of the initial configuration, as is well known for level-set methods (Allaire et al. 2004; Wang et al. 2003; Wang and Wang 2006). In Fig. 13 the objective history is plotted of the force inverter optimization problem. A smooth convergence is obtained, but many additional iterations are necessary to eliminate the unnecessary material. The changing topology and velocity field of this benchmark problem are shown in Fig. 14.

The second benchmark is a compliant gripper design problem solved with the same parameters as the compliant force inverter. The boundary conditions and the initial and optimal configuration are displayed in Fig. 15. Depending on the initial configuration, the optimal gripping configuration, very similar to the solution obtained

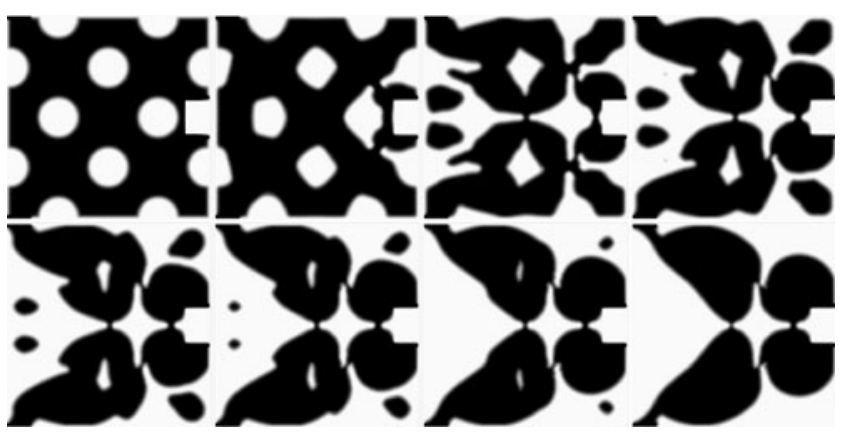

Fig. 16 Plot of the evolving element densities of a compliant gripper design problem using the level-set method combined with ECP

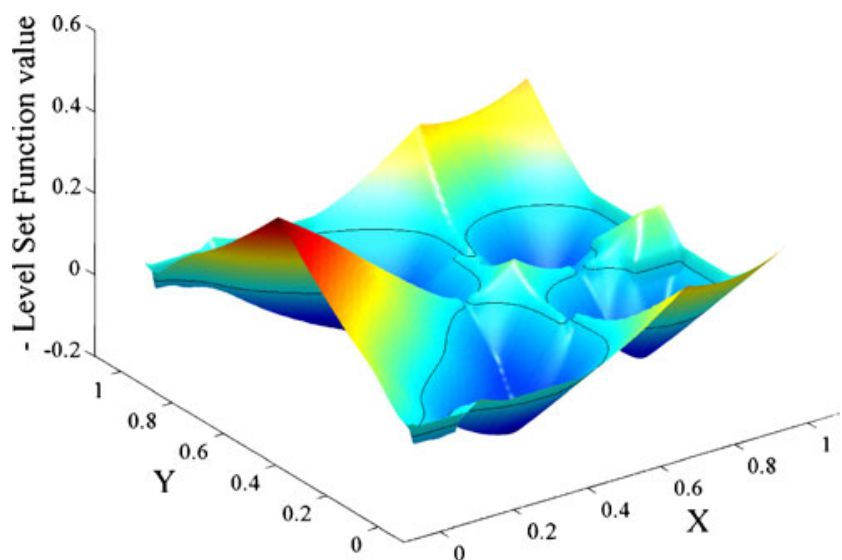

Fig. 17 The level-set function of the optimal configuration of a complaint gripper design problem using the level-set method combined with ECP

in Allaire et al. (2004), is also obtained. The evolution of the element densities of the compliant gripper design and the level-set function can be found in Figs. 16 and 17, respectively.

However, some problems involving the level-set formulation are observed. The optimized structures include thin hinge-like parts, which are sometimes lost at a certain moment in the optimization history. This surface-loss may be attributed to the usage of upwind schemes and an underresolved interpolation of the level-set function (Wang and Wang 2006). In some cases the compliant force inverter problem encountered a stability point in the structural analysis. Though the analysis is able to cope with this stability point, it causes large differences between the structural and adjoint displacements in two subsequent iterations of the optimization. Then oscillations around this stability point may hinder further convergence of the optimization process. However, these issues are unrelated to the proposed combination and may be solved by adding requirements to the design problem.

\section{Conclusions}

The components of a level-set method can be chosen from a variety of different approaches to obtain an efficient and practical new variant in the level-set method family. This work combines the element connectivity parameterization method (ECP) material interpolation has been used in a level-set framework.

The resulting topology optimization method exploits the crisp boundary, and manufacturability of the optimum designs of the level-set method and combines this with the ad vantages of ECP in the field of complex nonlinearities. The effectiveness has been demonstrated on some benchmark problems commonly used in compliance minimization and 
compliant mechanism design optimization. Some issues concerning the level-set method remain unsolved including the dependence of the final result on the initial configuration. However, this combination of methods offers a new perspective on a general approach to deal with nonlinearities in topology optimization ensuring a crisp boundary design.

Acknowledgement The authors would like to acknowledge the funding of the Dutch MicroNed programme which made this research possible.

Open Access This article is distributed under the terms of the Creative Commons Attribution Noncommercial License which permits any noncommercial use, distribution, and reproduction in any medium, provided the original author(s) and source are credited.

\section{Appendix}

The derivation of the 'naturally' extended velocity field and Lagrange multiplier estimate is given here.

\section{Structural minimization problem}

The structural minimization problem on a domain $\Omega$ with Dirichlet and Neumann boundary $\Gamma_{N}$ and $\Gamma_{D}$ with general objective $J(\mathbf{v})$ was given by,

$\min _{\Omega} J(\mathbf{v})=\int_{\Omega} j_{\Omega}(\mathbf{v}) d \Omega+\int_{\Gamma} j_{\Gamma}(\mathbf{v}) d \Gamma$

subject to $\left\{\begin{array}{ccc}\nabla \cdot \sigma(\mathbf{v})+\mathbf{b}=\mathbf{0} & \text { on } & \Omega \\ \boldsymbol{\sigma}(\mathbf{v}) \cdot \mathbf{n}=\mathbf{t} & \text { on } & \Gamma_{N} \\ \mathbf{v}=\mathbf{0} & \text { on } & \Gamma_{D},\end{array}\right.$

where $\mathbf{v}$ is the displacement, $\boldsymbol{\sigma}$ is the stress, $\mathbf{b}$ is the body force vector, $\mathbf{n}$ is the normal to the boundary and $\mathbf{t}$ is the boundary traction vector. An additional volume constraint can be added to the constraints formulated as,

$G(\Omega)=\int_{\Omega} d \Omega-V_{\max } \leq 0$,

where $V_{\max }$ is the maximum volume. We formulate a Lagrangian called $\mathscr{L}$ for this minimization problem, by varying (37) with a test function $\mathbf{q}$ and a variational problem as,

$$
\begin{aligned}
\mathscr{L}(\mathbf{v}, \mathbf{q}, l, \Omega)= & \int_{\Omega} j_{\Omega}(\mathbf{v}) d \Omega+\int_{\Gamma} j_{\Gamma}(\mathbf{v}) d \Gamma \\
& +\int_{\Omega}(\nabla \mathbf{q}: \sigma(\mathbf{v})-\mathbf{q} \cdot \mathbf{b}) d \Omega-\int_{\Gamma} \mathbf{q} \cdot \mathbf{t} d \Gamma \\
& +l\left(\int_{\Omega} d \Omega-V_{\max }\right),
\end{aligned}
$$

where $\mathbf{v} \in\left\{H^{1}(\Omega) \mid \mathbf{v}=\mathbf{0}\right.$ on $\left.\Gamma_{D}\right\}$ is the displacement, $\mathbf{q} \in\left\{H^{1}(\Omega) \mid \mathbf{q}=\mathbf{0}\right.$ on $\left.\Gamma_{D}\right\}$ is the Lagrange multiplier for the equilibrium equation or adjoint to the displacement, $l$ is the Lagrange multiplier estimate for the volume constraint and $\Omega$ and $\Gamma$ are the domain and boundary of the current structural domain, respectively. The variation of the Lagrangian can be written as,

$\partial \mathscr{L}=\partial_{\mathbf{v}} \mathscr{L}+\partial_{\mathbf{q}} \mathscr{L}+\partial_{l} \mathscr{L}+\partial_{\Omega} \mathscr{L}$

A stationary point of the Lagrangian $\partial \mathscr{L}\left(\mathbf{u}, \mathbf{p}, \lambda, \Omega^{\star}\right)=0$ indicates a local minimum of the objective satisfying the constraints. Due to the Lagrange multipliers this point will be a saddle point. When we are not at a stationary point, $(\mathbf{v}, \mathbf{q}, l, \Omega)$, we ensure that the first three terms in (40) disappear in order to satisfy the constraints. The terms that are unbounded from below have now been eliminated from (40) and we obtain,

$\mathscr{L}^{k+1}=\mathscr{L}^{k}+\partial \mathscr{L}=\mathscr{L}^{k}+\partial_{\Omega} \mathscr{L}$,

from which we can choose a steepest descent direction for $\partial \Omega$ such that the last term in this equation is negative. But first the unbounded terms are eliminated by solving a structural and an adjoint problem.

Adjoint displacement

The condition $\partial_{\mathbf{v}} \mathscr{L}=0 \forall \partial \mathbf{v}$ gives,

$$
\begin{aligned}
0=\partial_{\mathbf{v}} \mathscr{L}= & \int_{\Omega}\left[\partial_{\mathbf{v}} j_{\Omega}(\mathbf{v})+\nabla \mathbf{q}: \partial_{\mathbf{v}} \boldsymbol{\sigma}(\mathbf{v})\right] d \Omega \\
& +\int_{\Gamma_{N}} \partial_{\mathbf{v}} j_{\Gamma}(\mathbf{v}) d \Gamma \\
= & \cdots=\int_{\Omega}\left[\frac{\partial j_{\Omega}}{\partial \mathbf{v}}-\nabla \cdot\left(\frac{\partial \boldsymbol{\sigma}}{\partial \nabla \mathbf{v}}: \nabla \mathbf{q}\right)\right] \cdot \partial \mathbf{v} d \Omega \\
& +\int_{\Gamma_{N}} \partial \mathbf{v} \cdot\left[\frac{\partial \boldsymbol{\sigma}}{\partial \nabla \mathbf{v}}: \nabla \mathbf{q} \cdot \mathbf{n}+\frac{\partial j_{\Gamma}}{\partial \mathbf{v}}\right] d \Gamma,
\end{aligned}
$$

in which integration by parts, the divergence theorem and $\partial \mathbf{v}=\mathbf{0}$ on $\Gamma_{D}$ have been used. The term $\partial \boldsymbol{\sigma} / \partial \nabla \mathbf{v}$ is the tangent operator around the solution $\mathbf{v}, \partial \boldsymbol{\sigma} / \partial \nabla \mathbf{v}=\partial \boldsymbol{\sigma} / \partial \boldsymbol{\epsilon}(\mathbf{v})$ : $\partial \boldsymbol{\epsilon}(\mathbf{v}) / \partial \nabla \mathbf{v}$. From (42) a strong set of equations for $\mathbf{q}$ can be derived,

$$
\left\{\begin{array}{ccc}
\nabla \cdot(\partial \boldsymbol{\sigma} / \partial \nabla \mathbf{v}: \nabla \mathbf{q})-\partial j_{\Omega} / \partial \mathbf{v}=\mathbf{0} & \text { on } & \Omega \\
\partial \boldsymbol{\sigma} / \partial \nabla \mathbf{v}: \nabla \mathbf{q} \cdot \mathbf{n}=-\partial j_{\Gamma} / \partial \mathbf{v} & \text { on } & \Gamma_{N} \\
\mathbf{q}=\mathbf{0} & \text { on } & \Gamma_{D},
\end{array}\right.
$$

where the last boundary condition is added because of the condition $\mathbf{q} \in\left\{H^{1}(\Omega) \mid \mathbf{q}=\mathbf{0}\right.$ on $\left.\Gamma_{D}\right\}$. 
Structural displacement

The condition $\partial_{\mathbf{q}} \mathscr{L}=0 \forall \partial \mathbf{q}$ yields,

$$
\begin{aligned}
0=\partial_{\mathbf{q}} \mathscr{L}= & \int_{\Omega}\left[\partial_{\mathbf{q}} \nabla \mathbf{q}: \sigma(\mathbf{v})-\partial \mathbf{q} \cdot \mathbf{b}\right] d \Omega-\int_{\Gamma_{N}} \partial \mathbf{q} \cdot \mathbf{t} d \Gamma \\
= & \cdots=\int_{\Omega}-\partial \mathbf{q} \cdot[\nabla \cdot \boldsymbol{\sigma}(\mathbf{v})+\mathbf{b}] d \Omega \\
& +\int_{\Gamma_{N}} \partial \mathbf{q} \cdot[\boldsymbol{\sigma}(\mathbf{v}) \cdot \mathbf{n}-\mathbf{t}] d \Gamma
\end{aligned}
$$

in which again integration by parts, the divergence theorem and now the fact that $\partial \mathbf{q}=\mathbf{0}$ on $\Gamma_{D}$ has been used. From this equation the strong set of equations for $\mathbf{v}$ is deduced,

$$
\left\{\begin{array}{ccc}
\nabla \cdot \boldsymbol{\sigma}(\mathbf{v})+\mathbf{b}=\mathbf{0} & \text { on } & \Omega \\
\boldsymbol{\sigma}(\mathbf{v}) \cdot \mathbf{n}=\mathbf{t} & \text { on } & \Gamma_{N} \\
\mathbf{v}=\mathbf{0} & \text { on } & \Gamma_{D},
\end{array}\right.
$$

where also the last condition comes from the requirement $\mathbf{v} \in\left\{H^{1}(\Omega) \mid \mathbf{v}=\mathbf{0}\right.$ on $\left.\Gamma_{D}\right\}$. In principle for all choices of $\Omega \in D$ the conditions $\partial_{\mathbf{q}} \mathscr{L}=0 \forall \partial \mathbf{q}$ and $\partial_{\mathbf{v}} \mathscr{L}=0 \forall \partial \mathbf{v}$ can be satisfied exactly because $\mathbf{q}$ and $\mathbf{v}$ are solved directly from the systems (43) and (45).

\section{Volume constraint}

Finally, the condition $\partial_{l} \mathscr{L}=0 \forall \partial l$ simply results in,

$$
\int_{\Omega} d \Omega-V_{\max }=0 \quad \text { if } l>0 .
$$

This requirement directly restricts the feasible domains $\Omega$ and can in practice not be satisfied exactly. When we are at a local minimum $\left(\mathbf{u}, \mathbf{p}, \lambda, \Omega^{\star}\right)$ of our minimization problem, the variation of the Lagrangian with respect to a domain variation $\partial_{\Omega} \mathscr{L}=0 \forall \partial \Omega$ is satisfied. From this condition the Lagrange multiplier $\lambda$ can then be obtained. When we are at any other point $(\mathbf{v}, \mathbf{q}, l, \Omega)$ we can obtain a Lagrange multiplier estimate for instance from the least-squares satisfaction of this condition $\partial_{\Omega} \mathscr{L} \approx 0 \forall \partial \Omega$, as discussed in "Lagrange multiplier estimate".

\section{Domain variation}

We proceed to look further into the term $\partial_{\Omega} \mathscr{L}$, originating directly from a variation of the domain $\Omega$. According to Allaire et al. (2004) the changing material domain $\Omega_{\theta}$ can be described by an advection of the original domain $\Omega$ by a vector field $\boldsymbol{\theta}(\mathbf{x})$,

$$
\Omega_{\theta}=\{\mathbf{x}+\boldsymbol{\theta}(\mathbf{x}) \mid \mathbf{x} \in \Omega\} .
$$

The variation of an arbitrary functional $a \in H^{1}(\Omega)$ due to an infinitesimal shape change of the domain $\Omega$ depends on the changes of the boundary only. For a general functional, called $a(\Omega)$, defined as,

$$
\begin{aligned}
a(\Omega) & =\int_{\Omega} a_{\Omega}(\mathbf{x}) d \Omega+\int_{\Gamma} a_{\Gamma}(\mathbf{x}) d \Gamma \\
& =\int_{\Omega} a_{\Omega} d \Omega+\int_{\Omega} \nabla \cdot\left[a_{\Gamma} \mathbf{n}\right] d \Omega,
\end{aligned}
$$

the variation $\partial_{\Omega} a$ at $\Omega$ is defined as,

$$
\partial_{\Omega} a=\int_{\Gamma} a_{\Omega} \boldsymbol{\theta} \cdot \mathbf{n} d \Gamma+\int_{\Gamma}\left(\nabla \cdot\left[a_{\Gamma} \mathbf{n}\right]\right) \boldsymbol{\theta} \cdot \mathbf{n} d \Gamma .
$$

An infinitesimal shape change only depends on the convection in the direction normal to the boundary. Therefore we can define $\boldsymbol{\theta}=\theta_{\mathbf{n}} \mathbf{n}$ and $\boldsymbol{\theta} \cdot \mathbf{n}=\theta_{\mathbf{n}}$ without loss of generality. We end up with the relation,

$\partial_{\Omega} a=\int_{\Gamma} a_{\Omega} \theta_{\mathbf{n}} d \Gamma+\int_{\Gamma}\left(\nabla \cdot\left[a_{\Gamma} \mathbf{n}\right]\right) \theta_{\mathbf{n}} d \Gamma$.

Using this we can formulate the variation of the Lagrangian due to a domain change $\partial_{\Omega} \mathscr{L}=\partial_{\theta_{\mathbf{n}}} \mathscr{L}$ as,

$$
\begin{aligned}
\partial_{\Omega} \mathscr{L}=\int_{\Gamma}( & j_{\Omega}+\nabla \mathbf{q}: \boldsymbol{\sigma}(\mathbf{v})-\mathbf{q} \cdot \mathbf{b} \\
& \left.+\nabla \cdot\left[\left(j_{\Gamma}-\mathbf{q} \cdot \mathbf{t}\right) \mathbf{n}\right]+l\right) \theta_{\mathbf{n}} d \Gamma .
\end{aligned}
$$

Considering a non-design-dependent surface traction and no body force, we impose $\theta_{\mathbf{n}}=0$ on the part of the boundary where $\mathbf{t} \neq \mathbf{0}$ and set $\mathbf{b}=\mathbf{0}$. Defining $\Gamma_{0}=\Gamma \backslash \Gamma_{\mathbf{t} \neq \mathbf{0}}$, (51) reduces to,

$\partial_{\Omega} \mathscr{L}=\int_{\Gamma_{0}}\left(j_{\Omega}+\nabla \mathbf{q}: \sigma(\mathbf{v})+\nabla \cdot\left[j_{\Gamma} \mathbf{n}\right]+l\right) \theta_{\mathbf{n}} d \Gamma$.

\section{Lagrange multiplier estimate}

The stationarity condition of the Lagrangian $\partial_{\Omega} \mathscr{L}=0$, valid for any feasible boundary displacement $\theta_{\mathbf{n}}$ gives a relation for the Lagrange multiplier $\lambda$,

$j_{\Omega}+\nabla \mathbf{p}: \sigma(\mathbf{u})+\nabla \cdot\left[j_{\Gamma} \mathbf{n}\right]+\lambda=0 \quad$ on $\Gamma_{0}$.

For a given design with $\mathbf{v}$ and $\mathbf{q}$ the total error $\varepsilon$ in terms of the Lagrange multiplier estimate $l$ is given by,

$$
\begin{aligned}
\varepsilon(l) & =\int_{\Gamma_{0}}\left(j_{\Omega}+\nabla \mathbf{q}: \boldsymbol{\sigma}(\mathbf{v})+\nabla \cdot\left[j_{\Gamma} \mathbf{n}\right]\right) d \Gamma+l \int_{\Gamma_{0}} d \Gamma \\
& =\int_{\Gamma_{0}} \tilde{f} d \Gamma+l \int_{\Gamma_{0}} d \Gamma .
\end{aligned}
$$


Setting the derivative of the least-squares problem,

$$
\begin{aligned}
\min _{l} \varepsilon^{2}= & \left(\int_{\Gamma_{0}} \tilde{f} d \Gamma\right)^{2}+2 l \int_{\Gamma_{0}} \tilde{f} d \Gamma \cdot \int_{\Gamma_{0}} d \Gamma \\
& +l^{2}\left(\int_{\Gamma_{0}} d \Gamma\right)^{2},
\end{aligned}
$$

with respect to the Lagrange multiplier estimate $l$ equal to zero, gives the relation,

$l=-\frac{\int_{\Gamma_{0}}\left(j_{\Omega}+\nabla \mathbf{q}: \boldsymbol{\sigma}(\mathbf{v})+\nabla \cdot\left[j_{\Gamma} \mathbf{n}\right]\right) d \Gamma}{\int_{\Gamma_{0}} d \Gamma}$.

This Lagrange multiplier estimate is calculated when the constraint is active or otherwise set to zero. When the constraint is active, the calculated Lagrange multiplier estimate should be larger than zero, $l>0$.

\section{Steepest descent direction}

The steepest descent direction can be derived from (52). However, the active volume constraint cannot be satisfied exactly due to finite design changes and the Lagrange multiplier estimate $l$ only obstructs the optimization to deviate further from the feasible design space. Therefore, we can still end up with an infeasible solution.

In order to eliminate this problem, we penalize an infeasible solution to this system by formulating an augmented Lagrangian $\mathscr{L}_{\alpha}$ as,

$$
\begin{aligned}
\mathscr{L}_{\alpha}(\mathbf{v}, \mathbf{q}, l, \Omega)= & \int_{\Omega} j_{\Omega}(\mathbf{v}) d \Omega+\int_{\Gamma} j_{\Gamma}(\mathbf{v}) d \Gamma \\
& +\int_{\Omega}(\nabla \mathbf{q}: \sigma(\mathbf{v})-\mathbf{q} \cdot \mathbf{b}) d \Omega-\int_{\Gamma} \mathbf{q} \cdot \mathbf{t} d \Gamma \\
& +l\left(\int_{\Omega} d \Omega-V_{\max }\right) \\
& +\frac{\alpha}{2}\left(\int_{\Omega} d \Omega-V_{\max }\right)^{2}
\end{aligned}
$$

where $\alpha>0$ is a penalty parameter of arbitrary magnitude that should be zero when the volume constraint is inactive.

The Lagrange multiplier estimate $l$ is still solved from (56). The term $\partial_{\Omega} \mathscr{L}_{\alpha}$ now becomes,

$$
\begin{gathered}
\partial_{\Omega} \mathscr{L}_{\alpha}=\int_{\Gamma_{0}}\left(j_{\Omega}+\nabla \mathbf{q}: \boldsymbol{\sigma}(\mathbf{v})+\nabla \cdot\left[j_{\Gamma} \mathbf{n}\right]\right. \\
+l+\alpha G(\Omega)) \theta_{\mathbf{n}} d \Gamma .
\end{gathered}
$$

An appropriate choice for $\theta_{\mathbf{n}}$ ensures that $\partial_{\Omega} \mathscr{L}_{\alpha}<0$. The steepest descent choice gives,

$$
\begin{gathered}
\theta_{\mathbf{n}}=-s\left(j_{\Omega}+\nabla \mathbf{q}: \boldsymbol{\sigma}(\mathbf{v})+\nabla \cdot\left[j_{\Gamma} \mathbf{n}\right]\right. \\
+l+\alpha G(\Omega)) \quad \text { on } \Gamma_{0},
\end{gathered}
$$

where $s>0$ defines the magnitude of the shape change. We can now define the normal design boundary velocity field $v_{\mathbf{n}}$ on the whole domain as,

$$
\begin{gathered}
v_{\mathbf{n}}=-s\left(j_{\Omega}+\nabla \mathbf{q}: \sigma(\mathbf{v})+\nabla \cdot\left[j_{\Gamma} \mathbf{n}\right]\right. \\
+l+\alpha G(\Omega)) \text { on } \Omega .
\end{gathered}
$$

\section{References}

Allaire G, Jouve F, Toader AM (2004) Structural optimization using sensitivity analysis and a level-set method. J Comput Phys 194(1):363-393

Bruns TE, Tortorelli DA (2003) An element removal and reintroduction strategy for the topology optimization of structures and compliant mechanisms. Int J Numer Methods Eng 57(10):14131430

de Gournay F (2006) Velocity extension for the Level-set method and multiple eigenvalues in shape optimization. SIAM J Control Optim 45(1):343-367

Garreau S, Guillaume P, Masmoudi M (2001) The topological asymptotic for PDE systems: the elasticity case. SIAM J Control Optim 39(6): 1756-1778

Langelaar M (2006) Design optimization of shape memory alloy structures. PhD thesis at Delft University of Technology

Luo Z, Tong L (2008) A level set method for shape and topology optimization of large-displacement compliant mechanisms. Int J Numer Methods Eng 76(6):862-892. doi:10.1002/nme.2352

Sethian JA (1999) Level set methods and fast marching methods. Cambridge University Press, Cambridge

Sethian JA, Wiegmann A (2000) Structural boundary design via level set and immersed interface methods. J Comput Phys 163(2):489582(40)

Sokolowski J, Zochowski A (1999) On the topological derivative in shape optimization. SIAM J Control Optim 37(4):1251-1272

Wang S, Wang MY (2006) Radial basis functions and level set method for structural topology optimization. Int J Numer Methods Eng 65(12):2060-2090

Wang MY, Wang X, Guo D (2003) A level set method for structural topology optimization. Comput Methods Appl Mech Eng 192(1-2):227-246

Yoon GH, Kim YY (2005a) Element connectivity parameterization method for topology optimization of geometrically nonlinear structures. Int J Solids Struct 42(7):1983-2009

Yoon GH, Kim YY (2005b) The element connectivity parameterization formulation for the topology design optimization of multiphysics systems. Int J Numer Methods Eng 64(12):1649-1677

Yoon GH, Joung YS, Kim YY (2005) Optimal layout design using the element connectivity parameterization method: application to three-dimensional geometrical nonlinear structures. In: Sixth world congress of structural and multi-disciplinary optimization

Yoon GH, Kim YY, Langelaar M, van Keulen F (2008) Theoretical aspects of the internal element connectivity parameterization approach for topology optimization. Int J Numer Methods Eng 67:775-797. doi:10.1002/nme.2342 